\title{
Oral poly(ethylene glycol)-conjugated human recombinant lysozyme control of lung metastases in mice
}

\author{
MORENO COCCHIETTO ${ }^{1}$, LAURA ZORZIN ${ }^{1}$, PAOLO ALBERTO VERONESI ${ }^{3}$ and GIANNI SAVA ${ }^{1,2}$ \\ ${ }^{1}$ Callerio Foundation Onlus, Via Alexander Fleming 22-31; ${ }^{2}$ Department of Biomedical Sciences, University of Trieste, \\ Via Giorgieri 7, 34127 Trieste; ${ }^{3}$ Therapicon Srl, Via Marchesi de Taddei 21, 20146 Milan, Italy
}

Received May 23, 2008; Accepted July 23, 2008

DOI: $10.3892 / \mathrm{mmr} 00000039$

\begin{abstract}
Human recombinant lysozymes (rHLZs), particularly hen egg-white lysozyme (HEL), are promising agents for the treatment of diseases such as cancer. However, in a similar but improved fashion to what has already been demonstrated using HEL, the PEGylation of an rHLZ leads to a new drug that appears to protect against spontaneous lung metastasis development in mice bearing mammary carcinoma (MCa). The oral administration of $25-100 \mathrm{mg} / \mathrm{kg} /$ day of rHLZ-PEG (HEL-equivalent dose) to CBA female mice, admixed with daily food for 14 consecutive days, significantly reduced the growth of the primary tumour by up to $30 \%$ and of lung metastasis weight by up to $95 \%$, as compared to the untreated controls. An anti-metastatic effect significantly higher than that of uncoupled rHLZ was also confirmed in MCa-carrying animals immunosuppressed by cyclophosphamide or cyclosporine. This is of note if one takes into consideration the fact that virtually all chemotherapeutic regimens can cause immune system depression, with a consequent limitation of the dosage and effectiveness of anti-tumour treatments.
\end{abstract}

\section{Introduction}

Lysozyme (LZ), in particular hen egg-white lysozyme (HEL), is endowed with a number of biological properties, including anti-tumour and anti-metastatic activity (1-3). There is evidence to support the theory that the moderate LZ-induced reduction of tumours and the much more pronounced reduction of metastasis in models of rodent transplantable tumours are not associated with direct action on tumour cells, but are rather related to the elicitation of unspecific responses in the host $(2,4,5)$. Additionally, in combination with cisplatin or 5-fluorouracil, HEL has exhibited an immunoadjuvant and therapeutically synergic action in pre-clinical models $(1,6)$. This fact has interesting implications, considering that virtually all chemotherapeutic regimens can cause a degree of immune

Correspondence to: Dr Gianni Sava, Callerio Foundation Onlus, Via A. Fleming 22-31, 34127 Trieste, Italy

E-mail: g.sava@callerio.org

Key words: lysozymes, oral treatment, tumour system depression, limiting the applicable doses and the effectiveness of anti-cancer treatments (7-9). As well, tumours themselves produce a number of factors (i.e., cytokines) which may directly or indirectly inhibit immune response, blinding the immune system of the host to the presence of abnormal antigens and hampering immunotherapy $(10,11)$. Human LZ (HLZ) is one of the major anti-microbial proteins present in milk. Recently, its recombinant form (rHLZ) has started to be used as a protein model in biological studies (12). Previous results in a mouse model of solid metastatic tumour have shown that a PEGylated HEL is significantly more effective against the formation of metastases than native non-PEGylated HEL (13). It is well know that, among the processes used to increase the volume of the active molecule by slowing down renal filtration and increasing residence time in circulation, polyethylene glycol PEGylation is the most widely employed technology, increasing the clearance half-life time and the stability of drugs (14). The aim of the present study was to study in vivo the effects of the oral administration of a novel rHLZ (15) and its PEGylated form (rHLZ-PEG) (16) on tumour growth and metastasis development in mammary carcinoma (MCa)-bearing CBA mice (17). The effect of rHLZ-PEG was compared to that of HEL and of non-conjugated rHLZ. Furthermore, the anti-tumour and anti-metastatic effectiveness of rHLZ-PEG was evaluated in animals treated with conventional immunosuppressing doses of cyclosporine (CsA) or cyclophosphamide (CF).

\section{Materials and methods}

Drugs and doses. HEL (SPA, Milan; MW=14,400) was administered at a dose of $100 \mathrm{mg} / \mathrm{kg}$ per day, a dosage which has been proven as effective in a previous test (18). rHLZ (MW=16,537) and rHLZ-PEG (MW=34,520) were provided by Therapicon Srl (Milan, Italy). rHLZ was administered at HEL-equivalent doses of 25,50 and $100 \mathrm{mg} / \mathrm{kg}$ per day. rHLZ-PEG was administered at doses equimolar to those of rHLZ. All compounds were administered orally with daily food (a medicated powdered food containing the daily dose of each compound tested) for 14 consecutive days. CsA (Sandimmun $^{\circledR}$ ) and CF (Endoxan-Asta ${ }^{\circledR}$ ) were purchased from Novartis Farma S.p.A. (Origgio, VA, Italy), and from Asta Medica AG (Frankfurt, Germany), respectively. CF was administered intraperitoneally (i.p.) at a single immunosuppressive dose of $200 \mathrm{mg} / \mathrm{kg}$ a day before tumour implantation 


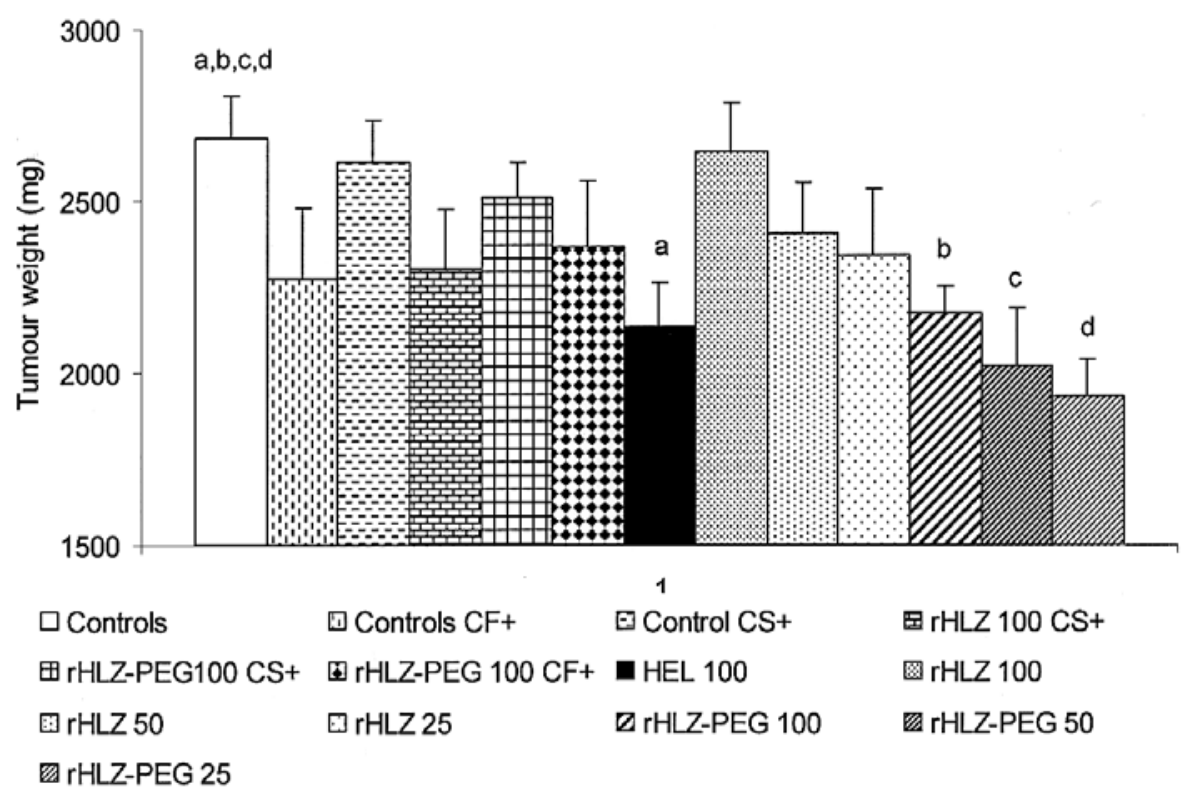

Figure 1. Effect of oral administration of lysozymes on tumour growth in mammary carcinoma (MCa)-bearing mice (with or without immunosuppression). Groups of eight CBA female mice implanted with $10^{6} \mathrm{MCa}$ cells on day 0 were orally-treated with $100 \mathrm{mg} / \mathrm{kg} \mathrm{HEL}, 25,50 \mathrm{and} 100 \mathrm{mg} / \mathrm{kg} \mathrm{rHLZ}$ (HEL equivalents) and rHLZ-PEG at doses equimolar to those of rHLZ. Compounds were admixed with daily powdered food for 14 consecutive days, starting on day 1. Immunosuppression was achieved with i.p. administration of $\mathrm{CF}$ at $200 \mathrm{mg} / \mathrm{kg}$, and of $\mathrm{CsA}$ at $50 \mathrm{mg} / \mathrm{kg} \mathrm{on} \mathrm{day} 1,25 \mathrm{mg} / \mathrm{kg}$ on days $1,4 \mathrm{and} 6$ and $10 \mathrm{mg} / \mathrm{kg}$ on days 8,11 and 13. Data refer to the final tumour measurement, taken on day 18. Values are expressed as the means \pm SE. Statistical analysis: Student-NewmanKeuls multiple comparison test. Groups with the same letter are statistically different. Statistaical significance: ${ }^{\mathrm{a}} \mathrm{p}<0.05,{ }^{\mathrm{b}-\mathrm{d}} \mathrm{p}<0.01$.

(day 1) (19). To acheive immunosuppression with CsA, a treatment protocol was adapted from that of Johnsson et al (20). CsA was administered i.p. at $50 \mathrm{mg} / \mathrm{kg}$ on day $1,25 \mathrm{mg} /$ $\mathrm{kg}$ on days 1,4 and 6 , and at $10 \mathrm{mg} / \mathrm{kg}$ on days 8,11 and 13 .

Animal model. CBA female mice (17-18 g) were used (Harlan Italy Srl, S. Pietro al Natisone, UD, Italy). Animal studies were carried out according to the guidelines in force in Italy (DDL 116, 21/2/1992 and subsequent addenda) and in a compliance with the Guide for the Care and Use of Laboratory Animals (21).

Solid tumour model. The murine MCa line used in the present study was derived from a spontaneous solid tumour in CBA female mice, subsequently isolated and stabilised at the Rudjer Boskovic Institute (Zagreb, Croatia) (17). The cell line is currently in use at the Callerio Foundation Onlus, where it is stored in cryogenic tanks with liquid nitrogen, in cryogenic vials containing $10^{7}$ viable cells of a single-cell suspension obtained from primary tumours at the third transplant generation. The tumour was resuscitated in vivo by intramuscular (i.m.) implantation of the contents of one vial of MCa cells taken from the cryogenic tank into the calf of the left hind leg of CBA female mice. After 14 days, the tumours of two mice ( $\sim 2 \mathrm{~g}$ of tumour mass) were harvested from the legs under sterile conditions (animals were sacrificed by cervical dislocation, the area of tumour growth was disinfected with an appropriate solution and animals were placed under a sterile box). A single-cell suspension was prepared: harvested tumors were pooled, minced with scissors, diluted with Dulbecco's phosphate-buffered saline ( $\mathrm{pH}$ 7.4) containing $\mathrm{Ca}^{+2}$ and $\mathrm{Mg}^{+2}$ (PBS) and filtered with a double layer of sterile gauze to remove tissue debris. The cell suspension was then centrifuged at $250 \mathrm{x} \mathrm{g}$ at $0-4^{\circ} \mathrm{C}$ for $10 \mathrm{~min}$. The supernatant was discarded while the pellet was re-suspended in an equal volume of PBS, and cell concentration and viability were determined by the trypan blue exclusion test with a Bürker's camera. Viable cells were finally diluted with PBS to the appropriate concentration required for implantation into the experimental animals, then re-implanted into four CBA female mice that constituted the donors for tumour propagation for experimental purposes.

Experimental protocol. Primary tumours were obtained by i.m. implantation of $10^{6}$ viable tumour cells into the calf of the left hind leg on day 0 . After tumour implantation, mice were randomly assigned to test groups of eight mice each. Primary tumour growth was evaluated with a calliper on days 7, 9, 11, 13,15 and 18 by measuring two orthogonal axes, and tumour weight was calculated using the formula: $(\pi / 6) \mathrm{x} \mathrm{a}^{2} \mathrm{x} b$, where $\mathrm{a}$ and $\mathrm{b}$ are the minor and major perpendicular axes, respectively. Data were then statistically analysed using the StudentNewmann-Keuls test. The number of lung metastases was evaluated on day 20 by counting the metastatic nodules per lung lobe under a low-power stereo microscope equipped with a graduated grid. The weight of the metastases was determined using the above formula.

\section{Results}

Primary tumour growth was reduced by rHLZ-PEG in a manner similar to growth reduction brought about by HEL, the standard used for reference. This reduction was also statistically significant at the lowest dose of rHLZ-PEG, of $25 \mathrm{mg} / \mathrm{kg} /$ day (Fig. 1). Lung metastases were reduced more significantly by rHLZ-PEG than by the corresponding unPEGylated molecule (Table I). Taken as a whole, the results obtained using rHLZ-PEG indicate an average reduction in metastasis number and weight of 48 and $85 \%$, respectively, 
Table I. Effect of different lysozymes on lung metastases.

\begin{tabular}{|c|c|c|c|c|}
\hline Group & No. & $\%$ (vs. controls) & Weight (mg) & $\%$ (vs. controls) \\
\hline Controls & $38 \pm 3^{a-c}$ & & $69.86 \pm 14.75^{\mathrm{d}-\mathrm{i}}$ & \\
\hline HEL 100 & $31 \pm 8$ & -18 & $41.76 \pm 28.69$ & -40 \\
\hline rHLZ 100 & $22 \pm 4$ & -42 & $8.73 \pm 3.71^{\mathrm{d}}$ & -87 \\
\hline rHLZ 50 & $30 \pm 6$ & -21 & $8.90 \pm 2.26^{\mathrm{e}}$ & -87 \\
\hline rHLZ 25 & $29 \pm 5$ & -23 & $27.06 \pm 9.12^{\mathrm{f}}$ & -61 \\
\hline rHLZ-PEG 100 & $22 \pm 3^{a}$ & -42 & $11.50 \pm 6.13^{\mathrm{g}}$ & -83 \\
\hline rHLZ-PEG 50 & $23 \pm 5^{b}$ & -39 & $16.71 \pm 6.78^{\mathrm{h}}$ & -76 \\
\hline rHLZ-PEG 25 & $14 \pm 4^{c}$ & -63 & $3.25 \pm 1.26^{\mathrm{i}}$ & -95 \\
\hline
\end{tabular}

Animals were sacrificed on day 20 and lung metastases were counted and measured. Each value is the mean \pm SE obtained from groups of eight mice. Statistical differences: means with the same letter are statistically different; Statistical significance: ${ }^{\mathrm{a}, \mathrm{b}, \mathrm{d}-\mathrm{h}} \mathrm{p}<0.05,{ }^{\mathrm{c}, \mathrm{i}} \mathrm{p}<0.01$.

Table II. Effect of treatment on lung metastases in immunosuppressed mice.

\begin{tabular}{llccc}
\hline Group & No. & $\%$ (vs. controls) & Weight (mg) & $\%$ (vs. controls) \\
\hline Controls & $38 \pm 3^{\mathrm{a}, \mathrm{b}}$ & & $69.86 \pm 14.75^{\mathrm{d}, \mathrm{e}}$ & -39 \\
Controls CF & $49 \pm 7^{\mathrm{c}}$ & +29 & $42.81 \pm 11.79^{\mathrm{f}}$ & $-79\left(\mathrm{CF}^{+}\right)$ \\
rHLZ-PEG 100 CF & $-55\left(\mathrm{CF}^{+}\right)$ & $5.32 \pm 5.49^{\mathrm{e}, \mathrm{f}}$ & -92 \\
Controls CS & $22 \pm 10^{\mathrm{c}}$ & -55 & $1.33 \pm 0.53^{\mathrm{d}}$ & $-75\left(\mathrm{CS}^{+}\right)$ \\
rHLZ-PEG 100 CS & $17 \pm 5^{\mathrm{a}}$ & $-41\left(\mathrm{CS}^{+}\right)$ & $10 \pm 2^{\mathrm{b}}$ &
\end{tabular}

Animals were sacrificed on day 20 and lung metastases were counted and measured. Each value is the mean \pm SE obtained from groups of eight mice. Statistical differences: means with the same letter are statistically different; Statistical significance: ${ }^{\mathrm{a}} \mathrm{p}<0.05 ;{ }^{\mathrm{b}, \mathrm{c}, \mathrm{f}} \mathrm{p}<0.01 ;{ }^{\mathrm{d}, \mathrm{e}} \mathrm{p}<0.001$.

as compared to the untreated controls (un-PEGylated rHLZ, 29 and $78 \%$; HEL, 18 and 40\%). rHLZ and rHLZ-PEG also significantly reduced lung metastasis formation in mice immunosuppressed with CF or CsA (Table II). Even when the schedule of CsA adopted for animal immuno-suppression significantly reduced metastasis formation per se, rHLZ-PEG further reduced the number and weight of lung metastases by an order of magnitude, independently of the immunosuppressor agent being used.

\section{Discussion}

Taken together, the data show the effectiveness of the oral administration of rHLZ coupled with PEG in controlling tumour and, in particular, metastasic growth in mouse MCa. Tumour and metastasis reduction after rHLZ-PEG administration occurred at HEL equivalent doses of $100 \mathrm{mg} / \mathrm{kg} /$ day, and also at two lower doses, showing a potency superior to that reported using PEGylated HEL in the same tumour model (13). Again similarly to past reports on HEL, rHLZ was equivalently or even more effective after PEGylation than in its uncoupled form. More notably, rHLZ-PEG is markedly effective in mice strongly immunosuppressed by CF or CsA. This indicates that rHLZ-PEG is capable of inhibiting metasta- tic growth, and that it plays a role in circumventing the effects of immunosuppression in treated animals. Considering that chemotherapy-induced immune system depression is often a dose-limiting major side effect of chemotherapeutic regimens, it could be argued that rHLZ-PEG would be beneficial in chemotherapeutic regimens by way of increasing the antitumour effects of anti-cancer drugs. Such synergic activity has already been demonstrated using HEL and a couple of antitumour drugs $(1,6)$.

The physical and chemical changes induced by PEGylation are known to increase many of the properties of the pharmacological behavior of the compound, such as the systemic retention of drugs, binding affinity and absorption and distribution patterns. We have previously reported that PEGylated HEL (HEL-PEG) is capable of reducing the number of ICAM-1+ and E-cadherin+ cells of the TS/A adenocarcinoma cell line in vitro, and to markedly prevent the formation of lung metastases in vivo in Balb/c and CBA mice models bearing i.m. implants of TS/A and MCa cells, respectively $(13,22)$. PEGylation might therefore confer new pharmacological properties to LZ, besides those related to its antibacterial activity and the modulation of host immunoresponses, such as the capacity to modify the ability of tumour cells to invade and metastasise. Indeed, it is known that ICAM-1 and 
E-cadherin are key regulators of the steps of tumour cell invasion, due to their role in cell-cell contacts (23).

What can be observed in the present investigation is that a recombinant human lysozyme is capable of controlling the growth of a malignant tumour in vivo. The differences seen between the effects of HEL and rHLZ (in particular HLZPEG) on primary tumours and on metastasis are in favour of rHLZ-PEG, and can be simply ascribed to differences between these two molecules, which are about $60 \%$ homologous (24). Nevertheless, the supposed mechanism of action for the antitumour activity of rHLZ should be the same as that of HEL, in that there is no doubt regarding their loss of enzymatic activity after PEGylation. This fact should exclude the participation of microbial peptidoglycan fragments with immunomodulating properties from the anti-tumour effects of the drugs (14). An understanding of the target of lysozymes and their PEGylated forms, which are responsible for a reduction in metastasic growth, could pave the way for the discovery of even more effective molecules for cancer treatment, which might be virtually free of side effects for the host.

\section{References}

1. Sava G, Perissin L and Zorzet S: Antimetastatic action of orally administered lysozyme in mice bearing Lewis lung carcinoma. Clin Exp Metastasis 6: 245-253, 1988.

2. Sava G, Ceschia V and Zabucchi G: Evidence for host-mediated antitumor effects of lysozyme in mice bearing the MCa mammary carinoma. Eur J Cancer Clin Oncol 24: 1737-1743, 1988.

3. Sava G, Benetti A, Ceschia V and Pacor S: Lysozyme and cancer: role of exogenous lysozyme as anticancer agent (review). Anticancer Res 9: 583-592, 1989.

4. Cartei F, Cartei G, Ceschia V, Pacor S and Sava G: Recovery of lymphocyte CD4+:CD8+ ratio in patients treated with lysozyme. Drug Invest 4: 51-57, 1992.

5. Pacor S, Giacomello E, Bergamo A, Gagliardi R, Cocchietto M and Sava G: Cytofluorimetric analysis of gut-intraepithelial and mesenteric lymph node lymphocytes of tumour bearing mice fed with egg-white lysozyme. Anticancer Res 16: 145-150, 1996.

6. Sava G, Pacor S, Dasic G and Bergamo A: Lysozyme stimulates lymphocyte response to ConA and IL-2 and potentiates 5fluorouracil action on advanced carcinomas. Anticancer Res 15: 1883-1888, 1995.
7. http://www.healthcastle.com/se_chemo.shtml

8. http://www.edinformatics.com/biotechnology/chemotherapy.htm

9. http://en.wikipedia.org/wiki/Chemotherapy

10. Wojtowicz-Praga S: Reversal of tumor-induced immunosuppression by TGF-beta inhibitors. Invest New Drug 21: 21-32, 2003.

11. Frumento G, Piazza T, Di Carlo E and Ferrini S: Targeting tumor-related immunosuppression for cancer immunotherapy. Endocr Metab Immune Disord Drug Targets 6: 233-237, 2006.

12. Yu Z, Meng Q, Yu H, et al: Expression and bioactivity of recombinant human lysozyme in the milk of transgenic mice. J Dairy Sci 89: 2911-2918, 2006.

13. Pacor S, Giacomello E, Bergamo A, et al: Antimetastatic action and lymphocyte activation by the modified lysozyme mPEGLyso in mice with MCa mammary carcinoma. Anticancer Res 16: 2559-2564, 1996.

14. Veronese FM and Pasut G: PEGylation, successful approach to drug delivery. Drug Discov Today 10: 1451-1458, 2005.

15. Huang J, Wu L, Yalda D, et al: Expression of functional recombinant human lysozyme in transgenic rice cell culture. Transgenic Res 11: 229-239, 2002.

16. http://www.patentstorm.us/patents/7012062-description.html

17. Poljak-Blazi M, Boranic M, Marzan B and Radacic M: A transplantable aplastic mammary carcinoma of CBA mice. Vet Arh 51: 99-107, 1981.

18. Sava G, Ceschia V, Pacor S and Zabucchi G: Observations on the antimetastatic action of lysozyme in mice bearing Lewis lung carcinoma. Anticancer Res 11: 1109-1114, 1991.

19. Tuve S, Chen B-M, Liu Y, et al: Combination of tumour sitelocated CTL-associated antigen-4 blockade and systemic regulatory $\mathrm{T}$-cell depletion induces tumour-destructive immune response. Cancer Res 67: 5929-5939, 2007.

21. Institute of Laboratory Animal Resources, Commission on Life Sciences, National Research Council: Guide for the Care and Use of Laboratory Animals. US Department of Health and Human Services, Public Health Service, National Institutes of Health, Bethesda, MD, DHHS Publication No. (NIH) 86-23, 1985.

21. Johnsson C, Andersson A, Bersztel A, Karlsson-Parra A, Gannedahl G and Tufveson G: Successful retrasplantation of mouse-to-rat cardiac xenografts under immunosuppressive monotherapy with cyclosporine. Transplantation 63: 652-656, 1997.

22. Pacor S, Gagliardi R, Di Daniel E, Vadori M and Sava G: In vitro regulation of ICAM-1 and E-cadherin and in vivo reduction of lung metastases of TS/A adenocarcinoma by lysozyme derivative. Int J Mol Med 4: 369-375, 1999.

23. Strumane $\mathrm{K}$, Bonnomet A, Stove $\mathrm{C}$, et al: E-cadherin regulates human Nanos1, which interacts with p120ctn and induces tumor cell migration and invasion. Cancer Res 66: 10007-10015, 2006.

24. http://linkinghub.elsevier.com/retrieve/pii/S0091674906028508 